Document downloaded from:

http://hdl.handle.net/10251/38332

This paper must be cited as:

Ponz Tienda, JL.; Pellicer Armiñana, E.; Yepes Piqueras, V. (2012). Complete fuzzy scheduling and fuzzy earned value management in construction projects. Journal of Zhejiang University Science A. 13(1):56-68. doi:10.1631/jzus.A1100160.

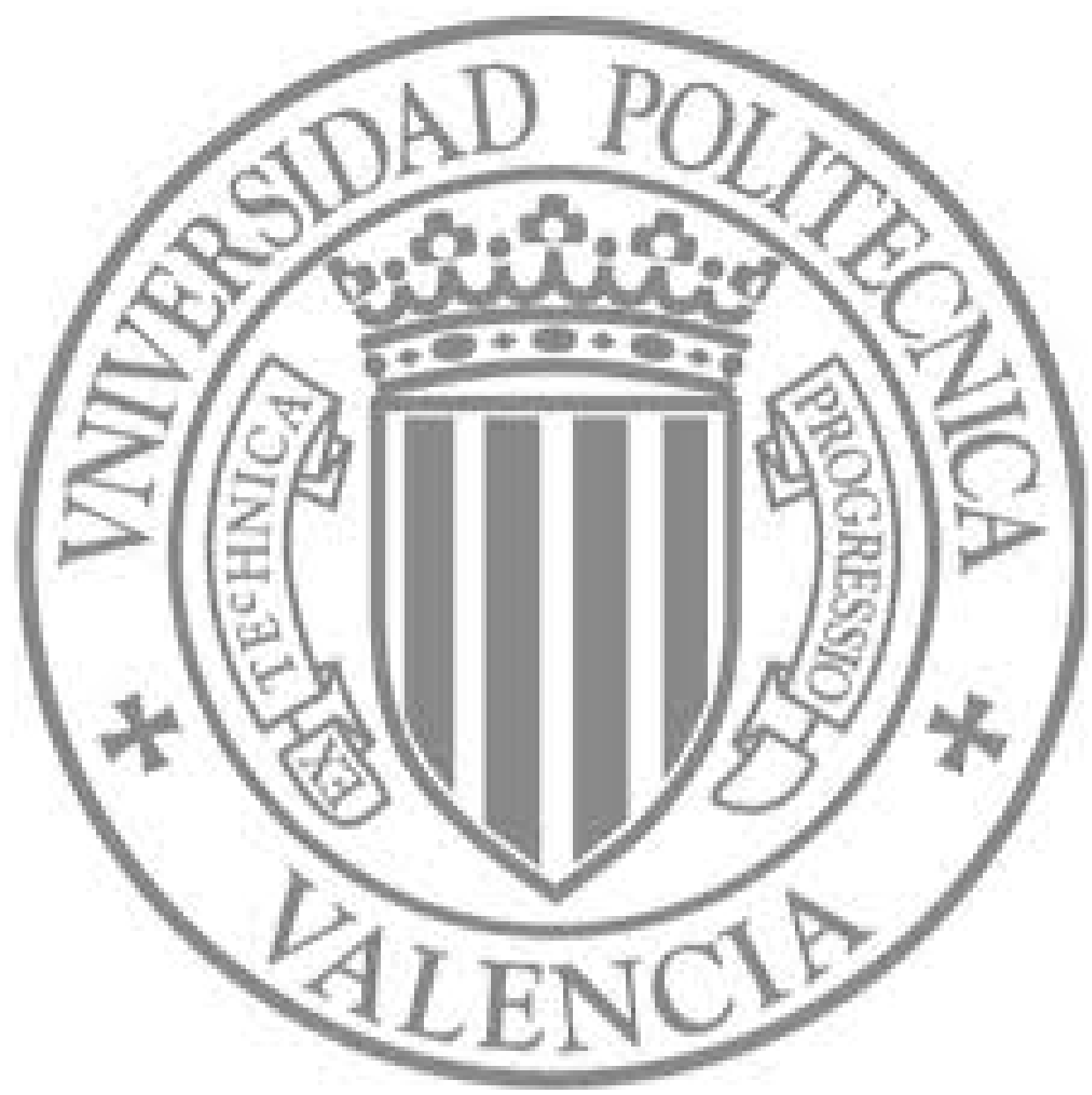

The final publication is available at

http://link.springer.com/article/10.1631\%2Fjzus.A1100160

Copyright Springer Verlag (Germany) 


\title{
Complete fuzzy scheduling and fuzzy earned value management in construction projects
}

\author{
José Luís PONZ-TIENDA ${ }^{1}$, Eugenio PELLICER ${ }^{2}$, Víctor YEPES ${ }^{\dagger * 3}$ \\ ( ${ }^{I}$ School of Building Engineering, Universitat Politècnica de València, 46022 Valencia, Spain) \\ ( ${ }^{2}$ School of Civil Engineering, Universitat Politècnica de València, 46022 Valencia, Spain) \\ $\left({ }^{3}\right.$ Department of Construction Engineering, ICITECH, Universitat Politècnica de València, 46022 Valencia, Spain) \\ †E-mail: vyepesp@upv.es \\ Received June 10, 2011; Revision accepted Sept. 7, 2011; Crosschecked Dec. 6, 2011
}

\begin{abstract}
This paper aims to present a comprehensive proposal for project scheduling and control by applying fuzzy earned value. It goes a step further than the existing literature: in the formulation of the fuzzy earned value we consider not only its duration, but also cost and production, and alternatives in the scheduling between the earliest and latest times. The mathematical model is implemented in a prototypical construction project with all the estimated values taken as fuzzy numbers. Our findings suggest that different possible schedules and the fuzzy arithmetic provide more objective results in uncertain environments than the traditional methodology. The proposed model allows for controlling the vagueness of the environment through the adjustment of the $\alpha$-cut, adapting it to the specific circumstances of the project.
\end{abstract}

Key words: Construction, Earned value method (EVM), Fuzzy logic, Fuzzy set, Project management, Scheduling doi:10.1631/jzus.A1100160 Document code: A CLC: TU71

\section{Introduction}

The goal of a construction project is the production of a unique product (prototype) under constraints of scope, time, and budget, with no more information than experience and research. To achieve its objectives, project performance must be observed and measured regularly to identify variances and establish corrective actions (Pellicer et al., 2009). The earned value method (EVM) is the best tool for measuring project progress, as it considers the interrelationship between production, costs and time in the project's forecasting to establish appropriate corrective measures (Fleming and Koppelman, 1998; Anbari, 2003). The EVM is basically a comparison between budgeted and scheduled with performed, obtaining dif-

\footnotetext{
Corresponding author

* Project partially supported by the Spanish Ministry of Science and Innovation (No. BIA2011-23602), and the European Community with the European Regional Development Fund (FEDER), Spain (C) Zhejiang University and Springer-Verlag Berlin Heidelberg 2012
}

ferent measures to report the progress of the project in terms of cost, production and time. Recently, this tool has been deeply analyzed and increasingly applied by practitioners, being expanded and improved with the earned schedule (ESch) (Lipke, 2003).

Scheduling offers alternatives between the earliest and latest execution times of the jobs, thus providing an unlimited number of different comparisons between feasible schedules; conclusions based on only one feasible schedule are not totally reliable. This fact is not considered in the traditional methodology, which also does not provide for the vagueness of the values used in the programming. In a real-world situation, activity durations in a project network may be difficult to define and estimate exactly (Chen and Huang, 2007). As a solution to the problem of this vagueness, alternatives were proposed based on uncertainty and imprecision (Lipke et al., 2009). Uncertainty refers to a random, statistical or probabilistic nature and imprecision to an incompletely defined nature. 
When the nature of the imprecision is not associated with the presence of random variables but to classes of membership, the theory of fuzzy sets (TFSs) may be applicable. The TFS facilitates the performance of any arithmetical operation with values that are not precisely known, but that can be limited within certain bounds of membership, including conditions of exclusion and tolerance, called fuzziness. The concept of a fuzzy set provided a theory to deal with incomplete or imprecise information (Zadeh, 1965). The TFS and probability are conceptually different. The TFS works with degrees of membership and with uncertainty, offering different models for the same real problems. There is a continuing and escalating debate about whether probability can only model particular types of uncertainty whereas the TFS is required to fill specific voids in the methods presently in use (Laviolette et al., 1995). Probability and the TFS are complementary rather than competitive (Zadeh, 1995), and they can live side by side in providing tools for uncertainty analysis in complex real world models.

Prade (1979) was the first researcher to use fuzzy numbers for scheduling. Further approaches were adopted in project scheduling problems by other authors. For example, Chanas and Kambourowski (1981) used fuzzy numbers to represent activity durations in project networks, changing the criticality concept computing the path degree of criticality. Chen and Huang (2007) developed a method to deal with completion time management and the critical degrees of all activities for a project network, representing the variable durations as linguistic variables of fuzzy numbers. Recently, new contributions have provided a clearer estimation of task finish times in fuzzy scheduling based on considerations of customer attributes (Liu et al., 2010).

Regarding the application of the TFS to the EVM, the first important contribution was presented by Kuchta (2005). Kuchta stated that the TFS allows for a more realistic project control related to risk management and improved the classical model for computing the values of estimate at completion (EAC), using fuzzy numbers instead of crisp numbers, using an easy-to-apply tool. Noori et al. (2008) introduced a novel approach based on fuzzy concepts to control schedule and cost in order to provide a better forecast, through a linguistic interpretation. They also proposed the use of fuzzy control charts based on $\alpha$-cuts with linguistic decisions and performance indexes divided into several parts. Finally, Moslemi et al. (2011) and Moslemi and Salehipour (2011) provided the most recent contribution to the fuzzy EVM. They incorporated the fuzzy principles into earned value (EV) calculations, developing a technique to measure and evaluate the performance and progress of a project and its activities under uncertainty, using linguistic terms for measuring the schedule and cost performance indexes (SPI/CPI), and introducing the problem of calculating the fuzzy ESch.

The purpose of our research was to go a step further than the analyzed literature, taking into consideration not only the duration, but also the cost and production in the formulation of the fuzzy EV. Therefore, this paper aims to present a robust and comprehensive proposal for project scheduling and control by applying the fuzzy EV. Fuzzy values are envisaged for the duration of the tasks, their costs and forecast production, as well as for alternatives in the scheduling between the earliest and latest times.

The remainder of this paper is organized as follows: Section 2 provides some basic notions of sets and fuzzy numbers. Section 3 details the algorithms for calculating the times of tasks using fuzzy values. Section 4 describes the method proposed for the fuzzy EV. The control measures are explained in Section 5. In Section 6, there is an implementation of the approach developed in a sample construction project. Finally, conclusions are drawn.

\section{Basic notions about fuzzy sets and fuzzy numbers}

To propose a notation that is consistent with all the existing literature is not possible. In this study we follow the notation put forward by Buckley et al. (2002).

A fuzzy set $\Omega$ is a grouping whose elements have different degrees of membership. Each possible individual has a value that represents its grade of similarity (membership) to the concept represented by the fuzzy set. Individuals in this universe (the set of entities) belong to the fuzzy set in different degrees of membership, represented by real numbers in the closed unit interval $[0,1]$. 
A triangular fuzzy number (Fig. 1 ) of a set $\Omega$ is a membership function written as $\tilde{A}(x)$ which produces values in $[0,1]$ for all $x$ in $\Omega$. It is defined by three numbers $a_{1} \leq a_{2} \leq a_{3}$, where the base of the triangle is the interval $\left[a_{1}, a_{3}\right]$ called the support (membership is equal to zero), and the vertex is $a_{2}$ (membership is equal to one).

$$
\widetilde{A}(x)=\left(a_{1}, a_{2}, a_{3}\right) .
$$

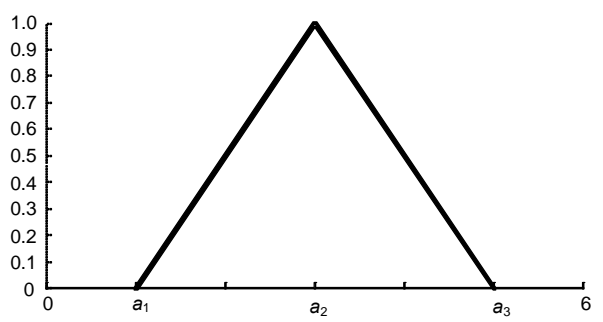

Fig. 1 Triangular fuzzy number

The fuzzy number shown in Fig. 1 could represent the duration, cost or quantity of production of a job, traditionally established by its value $a_{2}$ (crisp value). When there is enough information for a deterministic estimation, it is expressed in linguistic terms as "around $a_{2}$ ", with a membership between a minimum and maximum value $\left(a_{1}, a_{3}\right)$.

A trapezoidal fuzzy number (Fig. 2) of a set $\Omega$ is a membership function written as $\tilde{A}(x)$ which produces values in $[0,1]$ for all $x$ in $\Omega$ and is defined by four numbers $a_{1} \leq a_{2} \leq a_{3} \leq a_{4}$, where the base of the trapezoid is the interval $\left[a_{1}, a_{4}\right]$ (membership is equal to zero), and the top is over $\left[a_{2}, a_{3}\right]$ (membership is equal to one).

$$
\tilde{A}(x)=\left(a_{1}, a_{2}, a_{3}, a_{4}\right)
$$

$\alpha$-cuts of $\tilde{A}$, written as $\tilde{A}(\alpha)$, are slices through a fuzzy set that produce subsets in which one of its membership functions is greater than or equal to $\alpha$ (Fig. 3); for a triangular fuzzy number, $\widetilde{A}(\alpha)$ is obtained by applying Eq. (3). By changing the value of the $\alpha$-cut simultaneously to the fuzzy values of the duration, cost and production, the vagueness from an $\alpha$-cut $=0$ (which provides results with a maximum uncertainty) can be adjusted to an $\alpha$-cut $=1$, without any vagueness or ambiguity, and equivalent to the traditional deterministic methodology.

$$
\begin{gathered}
\widetilde{A}(\alpha)=\left(\operatorname{Si}(\alpha), a_{2}, \operatorname{Ss}(\alpha)\right) \\
\widetilde{A}(\alpha)=\left(a_{1}+\alpha\left(a_{2}-a_{1}\right), a_{2}, a_{2}+\alpha\left(a_{3}-a_{2}\right)\right) .
\end{gathered}
$$

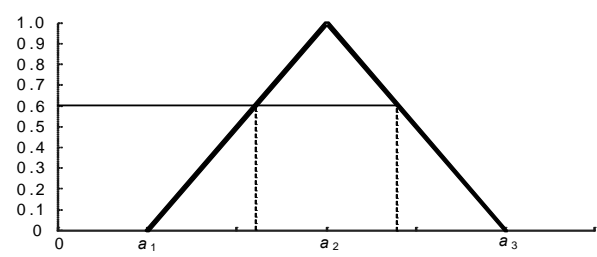

Fig. 2 Trapezoidal fuzzy number

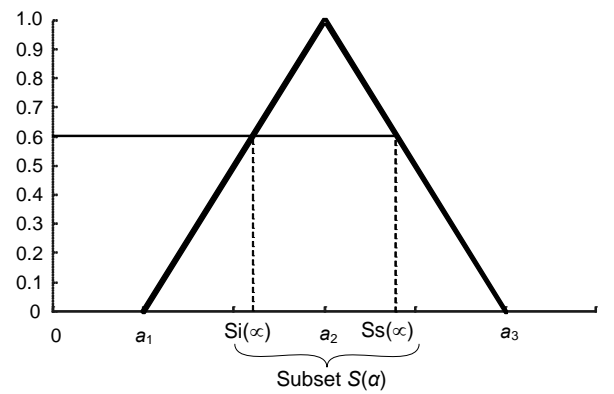

Fig. $3 \alpha$-cut $\widetilde{A}(\alpha)$ of $\widetilde{A}$

Basic fuzzy operations (represented as \#) between two triangular fuzzy numbers $\widetilde{A}$ and $\widetilde{B}$, are based on $\alpha$-cuts. They are defined in Eq. (4) for $0 \leq \alpha \leq 1$.

$$
\begin{aligned}
& \tilde{A}(\alpha) \# \widetilde{B}(\alpha)=\widetilde{C}(\alpha)=\left(c_{1}, c_{2}, c_{3}\right) \\
& \quad=\left\{\begin{array}{l}
c_{1}=\min \left(a_{1} \# b_{1}, a_{1} \# b_{3}, a_{3} \# b_{1}, a_{3} \# b_{3}\right), \\
c_{2}=a_{2} \# b_{2}, \\
c_{3}=\max \left(a_{1} \# b_{1}, a_{1} \# b_{3}, a_{3} \# b_{1}, a_{3} \# b_{3}\right) .
\end{array}\right.
\end{aligned}
$$

If $a_{1}, a_{2}, a_{3}, b_{1}, b_{2}, b_{3}>0$, then Eq. (4) can be simplified by applying Eq. (5).

$$
\begin{aligned}
& \widetilde{A}(\alpha) \oplus \widetilde{B}(\alpha)=\left(a_{1}+b_{1}, a_{2}+b_{2}, a_{3}+b_{3}\right), \\
& \widetilde{A}(\alpha) \ominus \widetilde{B}(\alpha)=\left(a_{1}-b_{3}, a_{2}-b_{2}, a_{3}-b_{1}\right), \\
& \widetilde{A}(\alpha) \otimes \widetilde{B}(\alpha)=\left(a_{1} \times b_{1}, a_{2} \times b_{2}, a_{3} \times b_{3}\right), \\
& \widetilde{A}(\alpha) \oslash \widetilde{B}(\alpha)=\left(a_{1} \div b_{3}, a_{2} \div b_{2}, a_{3} \div b_{1}\right) .
\end{aligned}
$$


To apply the algorithm described in Section 3, the fuzzy comparison rules must be defined (Yao and $\mathrm{Wu}, 2000)$. There are different methods of comparison, such as "strong comparison rules" and "weak comparison rules". While the "barycentric rule" (Fig. 4) is the most widely accepted rule for comparison between two triangular fuzzy numbers, some researchers prefer formulas that give more pessimistic results (Bonnai et al., 2004).

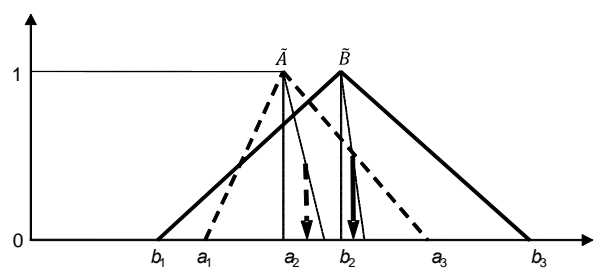

Fig. 4 Barycentric ordering

$$
\begin{aligned}
& \operatorname{Bar}(\tilde{A})=\frac{a_{1}+2 a_{2}+a_{3}}{4}, \\
& \operatorname{Bar}(\widetilde{B})=\frac{b_{1}+2 b_{2}+b_{3}}{4} .
\end{aligned}
$$

The barycentric rule obtained from Eq. (6) is outlined in Eq. (8).

$$
\text { if }\left\{\begin{array}{l}
\operatorname{Bar}(\widetilde{A})<\operatorname{Bar}(\widetilde{B}) \text { then } \widetilde{A}<\widetilde{B}, \\
\operatorname{Bar}(\widetilde{A})>\operatorname{Bar}(\widetilde{B}) \text { then } \widetilde{A}>\widetilde{B}, \\
\operatorname{Bar}(\widetilde{A})=\operatorname{Bar}(\widetilde{B}) \text {; then if }\left\{\begin{array}{l}
a_{3}>b_{3} \text { then } \widetilde{A}>\widetilde{B}, \\
a_{3}=b_{3} \text { and } a_{1}<b_{1} \text { then } \widetilde{A}<\widetilde{B}, \\
a_{3}<b_{3} \text { then } \widetilde{A}<\widetilde{B} .
\end{array}\right.
\end{array}\right.
$$

The methodology for deciding the values for limits is critical to the TFS. Basically, there are two kinds of computing techniques: Zadeh (1975)'s method based on the extension principles and Lawry (2001)'s method based on the mass assignment theory. Based on Zadeh (1975)'s method, Shipley et al. (1996) developed belief in fuzzy probability estimation of time (BIFPET) using human judgment instead of stochastic assumptions. From the extension principles, and three expert estimates with their respective fuzzy probability, the supervisor accepts or extends an additional degree of belief. In Lawry (2001)'s method, labels are assumed to be chosen from a finite predefined set of labels. The set of appropriate labels for a value is defined as a random set from a population of individuals in the subsets of labels. Wang and Hao (2007), using Zadeh (1975)'s method, and based on weighed generalized linguistics, provided a more solid foundation for scheduling in a fuzzy linguistic context, developing an easy and efficient technique.

\section{Project scheduling using fuzzy values}

The algorithm for calculating the times of the jobs in a project is very similar to that used in the traditional way. From a previous topologically ranked graph, the algorithm for calculating the earliest start $(\widetilde{\mathrm{ES}})$ and the earliest finish $(\widetilde{\mathrm{EF}})$ with finish-to-start precedence relations is stated in Eqs. (9) and (10).

Preliminary pass:

$$
\widetilde{\mathrm{ES}}_{\text {start }}=(0,0,0) \text {. }
$$

Forward pass:

$$
\begin{aligned}
& \text { For }(j=\text { start,finish, }+1), \\
& \begin{aligned}
\widetilde{\mathrm{ES}}_{j} & =\left(\mathrm{es}_{1 j}, \mathrm{es}_{2 j}, \mathrm{es}_{3 j}\right) \\
& =\widetilde{\max }\left(\widetilde{\mathrm{ES}}_{j}, \widetilde{\mathrm{EF}}_{i} \oplus \gamma_{i j}\right) ; \forall i \in P, \\
\widetilde{\mathrm{EF}}_{j} & =\left(\mathrm{ef}_{1 j}, \mathrm{ef}_{2 j}, \mathrm{ef}_{3 j}\right)=\widetilde{\mathrm{ES}}_{j} \oplus \widetilde{d}_{j},
\end{aligned}
\end{aligned}
$$

where $\tilde{d}_{j}$ is the fuzzy duration of a job $j, \gamma_{i j}$ the lag between $i$ and $j$, and $P$ the set of all the precedent jobs of $j$.

The latest start $(\widetilde{\mathrm{LS}})$ and latest finish $(\widetilde{\mathrm{LF}})$ fuzzy times will be computed by applying the backward pass algorithm expressed in Eqs. (11) and (12). Intermediate pass:

$$
\widetilde{\mathrm{LF}}_{\text {finish }}=\widetilde{\mathrm{EF}}_{\text {finish }} \text {. }
$$

Backward pass:

For $(i=$ finish,start, -1$)$,

$$
\begin{gathered}
\widetilde{\mathrm{LF}}_{i}=\left(\mathrm{lf}_{1 i}, \mathrm{lf}_{2 i}, \mathrm{lf}_{3 i}\right)=\widetilde{\min }\left(\widetilde{\mathrm{LF}}_{i}, \widetilde{\mathrm{LS}}_{j} \ominus \gamma_{i j}\right) ; \forall j \in S,(11) \\
\widetilde{\mathrm{LS}}_{i}=\left(\mathrm{ls}_{1 i}, \mathrm{ls}_{2 i}, \mathrm{ls}_{3 i}\right)=\widetilde{\mathrm{LF}}_{i}-\widetilde{d}_{i},
\end{gathered}
$$

where $S$ is the set of the successor jobs of $i$.

Note that in Eq. (12), the subtraction is a non-fuzzy operation (Ponz-Tienda, 2010), being 
computed as a fuzzy equation in which $\widetilde{\mathrm{LS}}_{j}$ is the variable to solve.

$$
\begin{gathered}
\widetilde{\mathrm{LS}}_{j} \oplus \tilde{d}_{j}=\widetilde{\mathrm{LF}}_{j}, \\
\widetilde{\mathrm{LS}}_{j}=\left(\mathrm{lf}_{1 j}-d_{1 j}, \mathrm{lf}_{2 j}-d_{2 j}, \mathrm{If}_{3 j}-d_{3 j}\right) .
\end{gathered}
$$

Once the above algorithm has been applied, the fuzzy Gantt chart will be obtained according to the earliest start times or according to the latest start times for each $\alpha$-cut (Fig. 5). The diagrams obtained are the upper and the lower bounds of all possible schedules. Obviously, there are a larger number of combinations between both situations, having an indeterminate number of possible expectations for project compliance.

\section{Earned value method using fuzzy values}

The three fundamental supports on which the EVM rests are the budgeted cost of work scheduled (BCWS), the actual cost of work performed (ACWP) and the budgeted cost of work performed (BCWP). The first two are equivalent to the forecast and what has been achieved, both in terms of cost and production, while the third is the real kernel of the EVM, on which the other elements are built.

\subsection{Budgeted cost of work scheduled}

The BCWS is a measure of the cost of work performed up to the status date or the current date, as the sum of the budgets of all the jobs of the project. It indicates how much of the budget cost should have been achieved, according to the project baseline dates.

The calculation of the fuzzy BCWS ( $\overline{\mathrm{BCWS}}$ ) will result from applying the expected fuzzy unit cost to the fuzzy forecasting production for each of the jobs in the project, obtaining as a result a non-triangular fuzzy value, due to the nonlinearity of the operation (Eq. (15)).

$$
\begin{aligned}
\widetilde{\operatorname{BCWS}}(\alpha) & =\sum_{i=1}^{N} \widetilde{\mathrm{bcws}}(\alpha)_{i}=\sum_{i=1}^{N} \widetilde{\mathrm{bc}}(\alpha)_{i} \otimes \widetilde{\mathrm{ws}}(\alpha)_{i} \\
& =\sum_{i=1}^{N}\left(\mathrm{bc}_{1 i} \times \mathrm{ws}_{1 i}, \mathrm{bc}_{2 i} \times \mathrm{ws}_{2 i}, \mathrm{bc}_{3 i} \times \mathrm{ws}_{3 i}\right),
\end{aligned}
$$

where $\widetilde{\operatorname{bcws}}(\alpha)_{i}$ is the fuzzy budgeted cost of work scheduled of each job obtained by the product of the fuzzy work scheduled $\left(\widetilde{\mathrm{ws}_{i}}\right)$ or forecasted production, with the fuzzy budgeted cost $\left(\widetilde{\mathrm{bc}_{i}}\right)$ or expected cost by production unit.

To distribute over time the $\overline{\mathrm{BCWS}}$ of the project, the fuzzy budgeted cost of work scheduled for each job must be divided by its duration and then applied from the starting date. Complexity arises when the BCWS, the dates and the durations are fuzzy numbers. Also, there is a countless diversity of possible dates for each of the jobs of the project, between its earliest and latest starting times.

The fuzzy BCWS at instant $t\left(\widehat{\operatorname{BCWS}}(\alpha)_{t}\right)$ should be calculated independently for each of the bounds scheduled. The scheduled earliest times

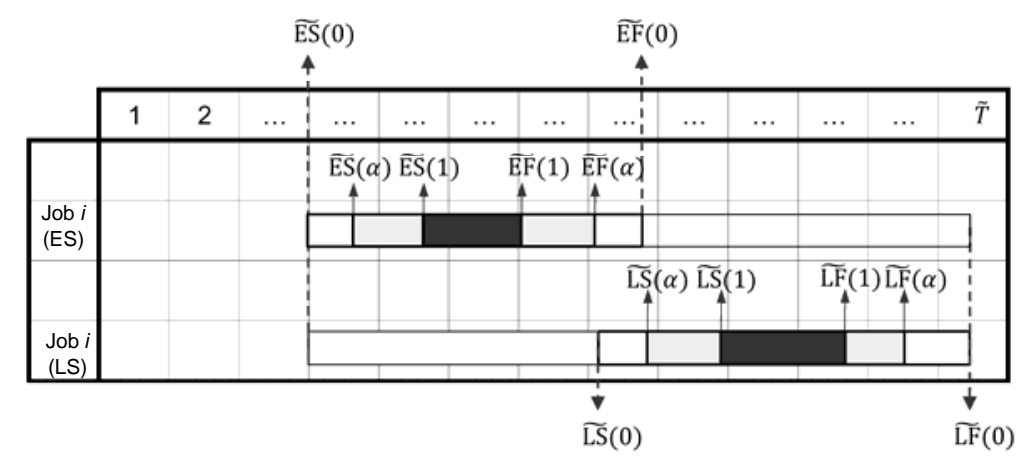

Fig. 5 Fuzzy Gantt chart according to the earliest and latest start times 
correspond to the left-hand side of $\overline{\mathrm{BCWS}}$, and later times to the right-hand side, taking care to operate as expressed in Eqs. (16) and (17).

$$
\begin{aligned}
& \overbrace{\overparen{\operatorname{BCWS}}(\alpha)_{t}}^{\text {es:Si }(\alpha)}=\sum_{1}^{t} \sum_{i=1}^{N}[\overbrace{\overbrace{\mathrm{bcws}}(\alpha)_{i t}}^{\mathrm{Si}(\alpha)} \div \overbrace{\tilde{d}(\alpha)_{i}}^{\mathrm{Si}(\alpha)}], \\
& \overbrace{\operatorname{\mathrm {BCWS}}(\alpha)_{t}}^{\mathrm{ss:Ss}(\alpha)}=\sum_{1}^{t} \sum_{i=1}^{N}[\overbrace{\overbrace{\mathrm{bcws}(\alpha)_{i t}}^{\mathrm{Ss}}(\alpha)} \div \overbrace{\tilde{d}(\alpha)_{i}}^{\mathrm{Ss}(\alpha)}] .
\end{aligned}
$$

Note that the division in Eqs. (16) and (17) is a non-fuzzy operation, being computed as expressed in Eq. (18).

$$
\left(\mathrm{bcws}_{1 i t} \div d_{1 i j}, \mathrm{bcws}_{2 i t} \div d_{2 i j}, \mathrm{bcws}_{3 i t} \div d_{3 i j}\right)
$$

The graphical interpretations of $\overline{\operatorname{BCWS}}(\alpha)_{t}$ and its fuzzy shape, applying Eqs. (16) and (17), are shown in Fig. 6.
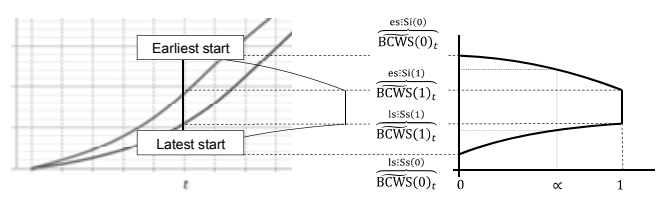

Fig. 6 Graphical interpretation of fuzzy $\mathrm{BCWS}_{t}$ at period $t$

For all values of $t$ greater than the planned makespan (mkp), the value of $\overline{\mathrm{BCWS}}$ at $t$ has to be considered, operating as expressed in Eq. (19).

$$
\forall t>\operatorname{mkp} ; \operatorname{BCWS}(\alpha)_{t}=\operatorname{BCWS}(\alpha)_{\mathrm{mkp}} \text {. }
$$

\subsection{Actual cost of work performed}

The ACWP is a crisp value, the result of multiplying the unit cost $\left(\mathrm{ac}_{i}\right)$ that is actually incurred for the work actually performed $\left(\mathrm{wp}_{i t}\right)$ in each of the periods and for each of the tasks of the project until the moment of control $t$ (Eq. (20)).

$$
\mathrm{ACWP}_{t}=\sum_{1}^{t} \sum_{i=1}^{N} \mathrm{ac}_{i} \times \mathrm{wp}_{i t} .
$$

\subsection{Budgeted cost of work performed}

The BCWP is known as the EV, and represents the value that the project should have at an instant $t$ in terms of budgeted cost. It is calculated by multiplying the budgeted cost by the work actually performed (Eq. (21)).

$$
\begin{aligned}
\widetilde{\operatorname{BCWP}}(\alpha)_{t} & =\sum_{1}^{t} \sum_{i=1}^{N} \widetilde{\mathrm{bc}}(\alpha)_{i} \times \mathrm{wp}_{i t} \\
& =\sum_{1}^{t} \sum_{i=1}^{N}\left(\mathrm{bc}_{1 i} \times \mathrm{wp}_{i t}, \mathrm{bc}_{2 i} \times \mathrm{wp}_{i t}, \mathrm{bc}_{3 i} \times \mathrm{wp}_{i t}\right) .
\end{aligned}
$$

\section{Measuring the progress of the project}

The EVM allows the measuring of efficiency in the progress and performance of a project's execution in terms of production costs and time simultaneously, by comparison with the programmed values. The three main indicators used to analyze the progress of the project are the schedule variation (SV), the cost variation $(\mathrm{CV})$ and the ESch.

\subsection{Schedule variation}

The SV is a measure of conformance of actual progress against the budgeted schedule in terms of production at instant $t$. It indicates the progress of production in units of expected cost (assuming that costs have not changed). It is calculated by the fuzzy difference between the $\overline{\mathrm{BCWP}}$ and $\overline{\mathrm{BCWS}}$ (Eq. (22) and Fig. 7).

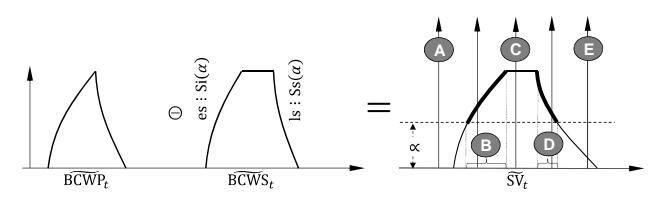

Fig. 7 Graphical interpretation of fuzzy schedule variation

$$
\begin{aligned}
& \widetilde{\mathrm{SV}}(\alpha)_{t}=\left(\mathrm{sv}_{1 t}, \mathrm{~Sv}_{2 t}, \mathrm{~Sv}_{3 t}, \mathrm{~Sv}_{4 t}\right) \\
& =\widetilde{\operatorname{BCWP}}(\alpha)_{t} \ominus \widetilde{\operatorname{BCWS}}(\alpha)_{t} \text {, } \\
& \mathrm{Sv}_{1 t}=\text { bcwp }_{1 t}-\text { bcws }_{4 t} \text {, } \\
& \mathrm{sv}_{2 t}=\text { bcwp }_{2 t}-\text { bcws }_{3 t} \text {, } \\
& \mathrm{sv}_{3 t}=\text { bcwp }_{2 t}-\text { bcws }_{2 t} \text {, } \\
& \mathrm{sv}_{4 t}=\text { bcwp }_{3 t}-\text { bcws }_{1 t} \text {. }
\end{aligned}
$$


There are five possible scenarios for the $\widetilde{\mathrm{SV}}$ results, depending on the sign and the $\alpha$-cut established (Table 1).

Table $1 \widetilde{\text { SV }}$ scenarios and linguistic interpretation

\begin{tabular}{|c|c|c|}
\hline $\begin{array}{c}\widetilde{\mathrm{SV}} \\
\text { scenario }\end{array}$ & Condition & $\begin{array}{c}\text { Linguistic } \\
\text { interpretation }\end{array}$ \\
\hline A & $\mathrm{SV}_{1 t, 2 t, 3 t, 4 t}>0$ & Ahead of schedule \\
\hline B & $\mathrm{sv}_{1 t} \leq \mathrm{Si}(\alpha) \wedge \mathrm{sv}_{2 t, 3 t, 4 t}>0$ & $\begin{array}{l}\text { Slightly ahead of } \\
\text { schedule }\end{array}$ \\
\hline $\mathrm{C}$ & $\mathrm{Sv}_{1 t, 2 t}<0 \wedge \mathrm{SV}_{3 t, 4 t}>0$ & On schedule \\
\hline $\mathrm{D}$ & $\mathrm{sv}_{1 t, 2 t, 3 t}<0 \wedge \mathrm{sv}_{4 t} \geq \mathrm{Ss}(\alpha)$ & $\begin{array}{l}\text { Slightly behind sched- } \\
\text { ule }\end{array}$ \\
\hline $\mathrm{E}$ & $\mathrm{SV}_{1 t, 2 t, 3 t, 4 t}<0$ & Behind schedule \\
\hline
\end{tabular}

It is important to note that when the control period is greater than the planned makespan, the SV measure fails, due to the absence of BCWP values from the planned makespan. To avoid this problem, all the values of $\mathrm{BCWS}_{t}$ for $t$ greater than the planned makespan must be established, by applying Eq. (19) (Fig. 12, page 10).

\subsection{Cost variation}

The CV is a measure of the budgetary conformance of ACWP at instant $t$. It is calculated as the fuzzy difference between the $\overline{\mathrm{BCWP}}$ and ACWP (Eq. (23) and Fig. 8).

$\widetilde{\mathrm{CV}}(\alpha)_{t}=\left(\mathrm{cv}_{1 t}, \mathrm{cv}_{2 t}, \mathrm{cv}_{3 t}\right)=\widetilde{\mathrm{BCWP}}(\alpha)_{t} \ominus \mathrm{ACWP}_{t}$, $\mathrm{cv}_{1 t}=\mathrm{bcwp}_{1 t}-\mathrm{ACWP}_{t}$,

$\mathrm{cv}_{2 t}=$ bcwp $_{2 t}-\mathrm{ACWP}_{t}$,

\subsection{Earned schedule}

The ESch is the time equivalent of the SV, projecting the $\mathrm{BCWP}_{p}$ on the $\mathrm{BCWS}$, measuring the schedule performance based on time and expressed in time units (Figs. 9 and 10).

Table $2 \widetilde{\mathrm{CV}}$ scenarios and linguistic interpretation

\begin{tabular}{cll}
\hline $\begin{array}{c}\widetilde{\mathrm{CV}} \\
\text { scenario }\end{array}$ & \multicolumn{1}{c}{ Condition } & \multicolumn{1}{c}{$\begin{array}{c}\text { Linguistic } \\
\text { interpretation }\end{array}$} \\
\hline $\mathrm{A}$ & $\mathrm{cv}_{1 t, 2 t, 3 t, 4 t}>0$ & Under budget \\
$\mathrm{B}$ & $\mathrm{cv}_{1 t}<0 \wedge \mathrm{Si}(\alpha) \geq 0$ & $\begin{array}{l}\text { Slightly under } \\
\text { budget }\end{array}$ \\
$\mathrm{C}$ & $\mathrm{cv}_{1 t} \leq 0 \wedge \mathrm{cv}_{3 t} \geq 0 \wedge \mathrm{cv}_{2 t} \in S(\alpha)$ & On budget \\
$\mathrm{D}$ & $\mathrm{cv}_{1 t, 2 t}<0 \wedge \mathrm{cv}_{3 t} \geq \mathrm{Ss}(\alpha)$ & Slightly over \\
$\mathrm{E}$ & $\mathrm{cv}_{1 t, 2 t, 3 t}<0$ & budget \\
\hline
\end{tabular}

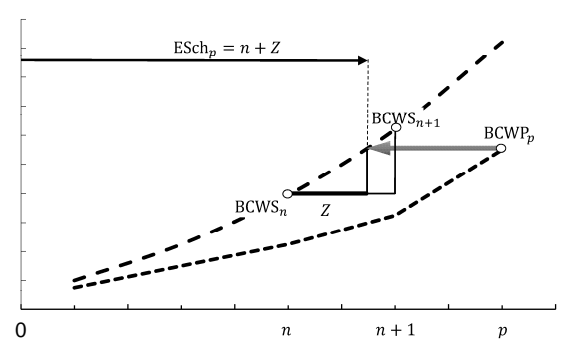

Fig. 9 Graphical representation of the earned schedule

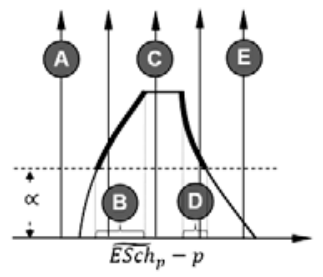

Fig. 10 Graphical interpretation of fuzzy earned schedule

The ESch is obtained by applying a triangular equivalence.

$Z=\left(\mathrm{BCWP}_{p}-\mathrm{BCWS}_{n}\right) /\left(\mathrm{BCWS}_{n+1}-\mathrm{BCWS}_{n}\right)$,

$\mathrm{ESch}_{p}=n+Z=n+\frac{\mathrm{BCWP}_{p}-\mathrm{BCWS}_{n}}{\mathrm{BCWS}_{n+1}-\mathrm{BCWS}_{n}}$.

Fig. 8 Graphical interpretation of fuzzy cost variation

There are five possible $\widetilde{\mathrm{CV}}$ scenarios, depending on the sign of the supports and the $\alpha$-cut established (Table 2).

The fuzzy operation for $\widetilde{\mathrm{ESch}}_{p}$ must be computed separately for its left-hand side (Eq. (25)) and right-hand side (Eq. (26)). 


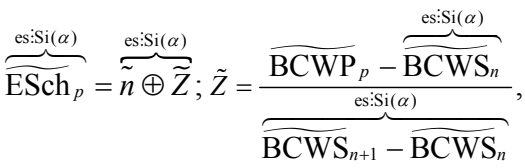

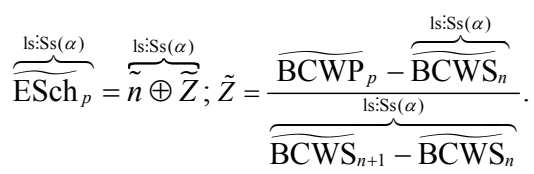

The five possible scenarios of the ESch $(\widetilde{\mathrm{ESch}})$ results, in linguistic terms are shown in Table 3.

Table $3 \widehat{\text { ESch }}$ scenarios and linguistic interpretation

\begin{tabular}{cll}
\hline $\begin{array}{c}\left(\widetilde{\mathrm{ESch}_{p}}-p\right) \\
\text { scenario }\end{array}$ & \multicolumn{1}{c}{ Condition } & \multicolumn{1}{c}{$\begin{array}{c}\text { Linguistic } \\
\text { interpretation }\end{array}$} \\
\hline $\mathrm{A}$ & $\mathrm{es}_{1 t, 2 t, 3 t, 4 t}>0$ & Ahead of time \\
$\mathrm{B}$ & $e s_{1 t} \leq S i(\alpha) \wedge \mathrm{es}_{2 t, 3 t, 4 t}>0$ & $\begin{array}{l}\text { Slightly ahead of } \\
\text { time }\end{array}$ \\
$\mathrm{C}$ & $e s_{1 t, 2 t}<0 \wedge \mathrm{es}_{3 t, 4 t}>0$ & On time \\
$\mathrm{D}$ & $e s_{1 t, 2 t, 3 t}<0 \wedge \mathrm{es}_{4 t} \geq S S(\alpha)$ & Slightly behind \\
$\mathrm{E}$ & $e s_{1 t, 2 t, 3 t, 4 t}<0$ & time \\
\hline
\end{tabular}

\section{Numerical experiment results}

In this section, a numerical experiment is described, implementing the approach developed in the fuzzy EVM. The example consists of a typical construction project that erects five complex units, each consisting of three processes indexed as process.unit (Fig. 11). The three processes are excavation, foundation and structure, and each unit comprises five identical houses. There is a unique team for each of the three processes.

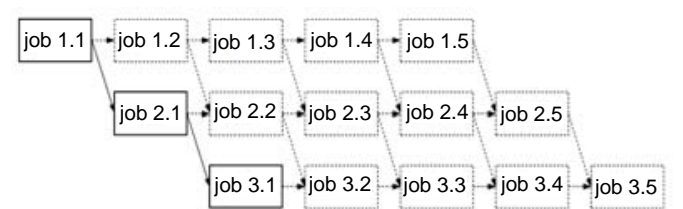

Fig. 11 Graph sample project

Fuzzy values of the planned production units, budgeted unit costs and estimated durations for the jobs are shown in Tables 4 and 5. In Table 4, the work schedules of processes 1 and 2 are measured in cubic meters, whereas that process 3 is measured in square meters, with $j$ being the repetitive units 1-5.

In Tables 4 and subsequently, $\operatorname{Si}(0)$ and $\operatorname{Ss}(0)$ are the supports of the fuzzy value, and $a_{2}$ the kernel or crisp value. Once the initial values are established, the fuzzy earliest start $(\widetilde{\mathrm{ES}}(0))$, earliest finish $(\widetilde{\mathrm{EF}}(0))$, latest start $(\widetilde{\mathrm{LS}}(0))$ and latest finish $(\widetilde{\mathrm{LF}}(0))$ times for each of the jobs are obtained by applying the algorithm described in Section 3.

Table 4 Planned fuzzy work budgeted fuzzy unit costs

\begin{tabular}{lrrrrrr}
\hline \multirow{2}{*}{ Job } & \multicolumn{3}{c}{$\widetilde{\mathrm{WS}_{i}}$} & \multicolumn{3}{c}{$\widetilde{\mathrm{bc}}_{i}$} \\
\cline { 2 - 7 } & \multicolumn{1}{c}{$\mathrm{Si}(0)$} & \multicolumn{1}{c}{$a_{2}$} & \multicolumn{1}{c}{$\mathrm{Ss}(0)$} & $\mathrm{Si}(0)$ & \multicolumn{1}{c}{$a_{2}$} & \multicolumn{1}{c}{$\mathrm{Ss}(0)$} \\
\hline $1-j$ & 1100.00 & 1150.00 & 1275.00 & 5.80 & 6.00 & 6.50 \\
$2-j$ & 120.00 & 125.00 & 135.00 & 14.50 & 15.00 & 15.75 \\
$3-j$ & 725.00 & 750.00 & 755.00 & 58.25 & 60.00 & 61.50 \\
\hline
\end{tabular}

Table 5 Estimated fuzzy durations

\begin{tabular}{lccc}
\hline \multirow{2}{*}{ Job } & \multicolumn{3}{c}{$\tilde{d}_{\boldsymbol{i}}$} \\
\cline { 2 - 4 } & $\operatorname{Si}(0)$ & $a_{2}$ & $\mathrm{Ss}(0)$ \\
\hline $1.1-1.2$ & 9 & 10 & 13 \\
1.3 & 9 & 10 & 12 \\
$1.4-1.5$ & 9 & 10 & 11 \\
2.1 & 14 & 15 & 17 \\
2.2 & 14 & 15 & 16 \\
$2.3-2.4-2.5$ & 13 & 15 & 16 \\
3.1 & 19 & 20 & 23 \\
3.2 & 19 & 20 & 22 \\
$3.3-3.4-3.5$ & 18 & 20 & 21 \\
\hline
\end{tabular}

The values of $\widetilde{\mathrm{bcws}_{i}}$ are expressed in monetary units, and $\overline{\mathrm{BCWS}}$ must be computed for each side of the shape and for all the jobs and $\alpha$-cuts (from 0 to 1 in step of 0.1 ) to determine the correct shape of the fuzzy number (Tables 7 and 8). The $\overline{\mathrm{BCWS}}(\alpha)$ of the project is obtained as the sum of each of the $\alpha$-cut values of all the $\widetilde{\operatorname{bcws}_{i}}(\alpha)$.

The $S$-curves of $\overline{\mathrm{BCWS}}$ are obtained by immediately applying Eqs. (16) and (17). For $\alpha$-cut $=0.7$, the values can be seen in Fig. 12. Note that the initial and final values correspond to triangular, and the intermediate shape to trapezoidal fuzzy numbers, due to the float of jobs, increasing the vagueness of supports as the forecast period moves away. 
For the control of the sample project, January 31 was set as the date for the beginning of the construction project, with a working week of five days and official holidays of the city of Valencia, Spain. Controls were to take place on the 15th and the last day of each month, reflecting for each of the jobs the production carried out to date and the costs incurred in its implementation. Once the established controls were processed, the results for the $\mathrm{CV}$ and the $\mathrm{SV}$ were as shown in Table 9. The interpretation of the previous results in linguistic terms is outlined in Table 10.

In Table 9, schedule variation $(\widetilde{\mathrm{SV}}(0.7))$ has two columns named $\left(a_{2}, a_{3}\right)$, being the core of the fuzzy numbers (as a result of the differences between the earliest and the latest times), and the lower $(\operatorname{Si}(0.7))$ and upper $(\mathrm{Ss}(0.7))$ limits of allowed deviation in accordance with the established $\alpha$-cut. The values of the column $a_{2}$ (exactly the same as offered by the

Table 6 Fuzzy scheduled times

\begin{tabular}{|c|c|c|c|c|c|c|c|c|c|c|c|c|}
\hline \multirow{2}{*}{ Job } & \multicolumn{3}{|c|}{$\widetilde{\mathrm{ES}}_{i}$} & \multicolumn{3}{|c|}{$\widetilde{E F}_{i}$} & \multicolumn{3}{|c|}{$\widetilde{\mathrm{LS}}_{i}$} & \multicolumn{3}{|c|}{$\widetilde{\mathrm{LF}}_{i}$} \\
\hline & $\operatorname{Si}(0)$ & $a_{2}$ & $\mathrm{Ss}(0)$ & $\mathrm{Si}(0)$ & $a_{2}$ & $\mathrm{Ss}(0)$ & $\mathrm{Si}(0)$ & $a_{2}$ & $\mathrm{Ss}(0)$ & $\operatorname{Si}(0)$ & $a_{2}$ & $\mathrm{Ss}(0)$ \\
\hline 1.1 & 0 & 0 & 0 & 9 & 10 & 13 & 0 & 0 & 0 & 10 & 10 & 13 \\
\hline 1.2 & 9 & 10 & 13 & 18 & 20 & 26 & 19 & 20 & 24 & 30 & 30 & 37 \\
\hline 1.3 & 18 & 20 & 26 & 27 & 30 & 38 & 39 & 40 & 47 & 50 & 50 & 59 \\
\hline 1.4 & 27 & 30 & 38 & 36 & 40 & 49 & 57 & 60 & 69 & 70 & 70 & 80 \\
\hline 1.5 & 36 & 40 & 49 & 45 & 50 & 60 & 75 & 80 & 90 & 90 & 90 & 101 \\
\hline 2.1 & 9 & 10 & 13 & 23 & 25 & 30 & 9 & 10 & 13 & 25 & 25 & 30 \\
\hline 2.2 & 23 & 25 & 30 & 37 & 40 & 46 & 28 & 30 & 37 & 45 & 45 & 53 \\
\hline 2.3 & 37 & 40 & 46 & 50 & 55 & 62 & 48 & 50 & 59 & 65 & 65 & 75 \\
\hline 2.4 & 50 & 55 & 62 & 63 & 70 & 78 & 66 & 70 & 80 & 85 & 85 & 96 \\
\hline 2.5 & 63 & 70 & 78 & 76 & 85 & 94 & 84 & 90 & 101 & 105 & 105 & 117 \\
\hline 3.1 & 23 & 25 & 30 & 42 & 45 & 53 & 23 & 25 & 30 & 45 & 45 & 53 \\
\hline 3.2 & 42 & 45 & 53 & 61 & 65 & 75 & 42 & 45 & 53 & 65 & 65 & 75 \\
\hline 3.3 & 61 & 65 & 75 & 79 & 85 & 96 & 61 & 65 & 75 & 85 & 85 & 96 \\
\hline 3.4 & 79 & 85 & 96 & 97 & 105 & 117 & 79 & 85 & 96 & 105 & 105 & 117 \\
\hline 3.5 & 97 & 105 & 117 & 115 & 125 & 138 & 97 & 105 & 117 & 125 & 125 & 138 \\
\hline
\end{tabular}

Table 7 Left-hand side of the $\widehat{\operatorname{bcws}}(\alpha)_{i}$ and $\widehat{\operatorname{BCWS}}(\alpha)$ for different $\alpha$-cuts

\begin{tabular}{|c|c|c|c|c|c|c|c|c|c|c|c|}
\hline \multirow{2}{*}{ Job } & \multicolumn{11}{|c|}{$\widetilde{\mathrm{bcws}}(\alpha)_{i}=\widetilde{\mathrm{bc}}(\alpha)_{i} \otimes \widetilde{\mathrm{ws}}(\alpha)_{i}$} \\
\hline & $\alpha=0$ & $\alpha=0.1$ & $\alpha=0.2$ & $\alpha=0.3$ & $\alpha=0.4$ & $\alpha=0.5$ & $\alpha=0.6$ & $\alpha=0.7$ & $\alpha=0.8$ & $\alpha=0.9$ & $\alpha=1.0$ \\
\hline 1.1 & 6380.0 & 6431.1 & 6482.4 & 6533.9 & 6585.6 & 6637.5 & 6689.6 & 6741.9 & 6794.4 & 6847.1 & 6900.0 \\
\hline 1.2 & 6380.0 & 6431.1 & 6482.4 & 6533.9 & 6585.6 & 6637.5 & 6689.6 & 6741.9 & 6794.4 & 6847.1 & 6900.0 \\
\hline 1.3 & 6380.0 & 6431.1 & 6482.4 & 6533.9 & 6585.6 & 6637.5 & 6689.6 & 6741.9 & 6794.4 & 6847.1 & 6900.0 \\
\hline 1.4 & 6380.0 & 6431.1 & 6482.4 & 6533.9 & 6585.6 & 6637.5 & 6689.6 & 6741.9 & 6794.4 & 6847.1 & 6900.0 \\
\hline 1.5 & 6380.0 & 6431.1 & 6482.4 & 6533.9 & 6585.6 & 6637.5 & 6689.6 & 6741.9 & 6794.4 & 6847.1 & 6900.0 \\
\hline 2.1 & 1740.0 & 1753.3 & 1766.6 & 1780.0 & 1793.4 & 1806.9 & 1820.4 & 1834.0 & 1847.6 & 1861.3 & 1875.0 \\
\hline 2.2 & 1740.0 & 1753.3 & 1766.6 & 1780.0 & 1793.4 & 1806.9 & 1820.4 & 1834.0 & 1847.6 & 1861.3 & 1875.0 \\
\hline 2.3 & 1740.0 & 1753.3 & 1766.6 & 1780.0 & 1793.4 & 1806.9 & 1820.4 & 1834.0 & 1847.6 & 1861.3 & 1875.0 \\
\hline 2.4 & 1740.0 & 1753.3 & 1766.6 & 1780.0 & 1793.4 & 1806.9 & 1820.4 & 1834.0 & 1847.6 & 1861.3 & 1875.0 \\
\hline 2.5 & 1740.0 & 1753.3 & 1766.6 & 1780.0 & 1793.4 & 1806.9 & 1820.4 & 1834.0 & 1847.6 & 1861.3 & 1875.0 \\
\hline 3.1 & 42231.3 & 42504.2 & 42778.0 & 43052.7 & 43328.3 & 43604.7 & 43882.0 & 44160.2 & 44439.3 & 44719.2 & 45000.0 \\
\hline 3.2 & 42231.3 & 42504.2 & 42778.0 & 43052.7 & 43328.3 & 43604.7 & 43882.0 & 44160.2 & 44439.3 & 44719.2 & 45000.0 \\
\hline 3.3 & 42231.3 & 42504.2 & 42778.0 & 43052.7 & 43328.3 & 43604.7 & 43882.0 & 44160.2 & 44439.3 & 44719.2 & 45000.0 \\
\hline 3.4 & 42231.3 & 42504.2 & 42778.0 & 43052.7 & 43328.3 & 43604.7 & 43882.0 & 44160.2 & 44439.3 & 44719.2 & 45000.0 \\
\hline 3.5 & 42231.3 & 42504.2 & 42778.0 & 43052.7 & 43328.3 & 43604.7 & 43882.0 & 44160.2 & 44439.3 & 44719.2 & 45000.0 \\
\hline$\widehat{\operatorname{BCWS}}(\alpha)$ & 20170 & 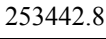 & 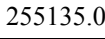 & & - & 200270 & 2 & 263680 & 265406.3 & 267137.8 & 268875.0 \\
\hline
\end{tabular}


Table 8 Right-hand side of the $\widehat{\operatorname{bcws}}(\alpha)_{i}$ and $\widehat{\operatorname{BCWS}}(\alpha)$ for different $\alpha$-cuts

\begin{tabular}{crrrrrrrrrrr}
\hline \multirow{2}{*}{ Job } & \multicolumn{10}{c}{$\widetilde{c} \widetilde{\mathrm{bcws}}(\alpha)_{i}=\widetilde{\mathrm{bc}}(\alpha)_{i} \otimes \widetilde{\mathrm{ws}}(\alpha)_{i}$} \\
\cline { 2 - 12 } & $\alpha=1$ & $\alpha=0.9$ & $\alpha=0.8$ & $\alpha=0.7$ & $\alpha=0.6$ & $\alpha=0.5$ & $\alpha=0.4$ & $\alpha=0.3$ & $\alpha=0.2$ & $\alpha=0.1$ & $\alpha=0.0$ \\
\hline 1.1 & 6900.0 & 7033.1 & 7167.5 & 7303.1 & 7440.0 & 7578.1 & 7717.5 & 7858.1 & 8000.0 & 8143.1 & 8287.5 \\
1.2 & 6900.0 & 7033.1 & 7167.5 & 7303.1 & 7440.0 & 7578.1 & 7717.5 & 7858.1 & 8000.0 & 8143.1 & 8287.5 \\
1.3 & 6900.0 & 7033.1 & 7167.5 & 7303.1 & 7440.0 & 7578.1 & 7717.5 & 7858.1 & 8000.0 & 8143.1 & 8287.5 \\
1.4 & 6900.0 & 7033.1 & 7167.5 & 7303.1 & 7440.0 & 7578.1 & 7717.5 & 7858.1 & 8000.0 & 8143.1 & 8287.5 \\
1.5 & 6900.0 & 7033.1 & 7167.5 & 7303.1 & 7440.0 & 7578.1 & 7717.5 & 7858.1 & 8000.0 & 8143.1 & 8287.5 \\
2.1 & 1875.0 & 1899.5 & 1924.1 & 1948.8 & 1973.7 & 1998.8 & 2024.0 & 2049.3 & 2074.8 & 2100.5 & 2126.3 \\
2.2 & 1875.0 & 1899.5 & 1924.1 & 1948.8 & 1973.7 & 1998.8 & 2024.0 & 2049.3 & 2074.8 & 2100.5 & 2126.3 \\
2.3 & 1875.0 & 1899.5 & 1924.1 & 1948.8 & 1973.7 & 1998.8 & 2024.0 & 2049.3 & 2074.8 & 2100.5 & 2126.3 \\
2.4 & 1875.0 & 1899.5 & 1924.1 & 1948.8 & 1973.7 & 1998.8 & 2024.0 & 2049.3 & 2074.8 & 2100.5 & 2126.3 \\
2.5 & 1875.0 & 1899.5 & 1924.1 & 1948.8 & 1973.7 & 1998.8 & 2024.0 & 2049.3 & 2074.8 & 2100.5 & 2126.3 \\
3.1 & 45000.0 & 45142.6 & 45285.3 & 45428.2 & 45571.2 & 45714.4 & 45857.7 & 46001.2 & 46144.8 & 46288.6 & 46432.5 \\
3.2 & 45000.0 & 45142.6 & 45285.3 & 45428.2 & 45571.2 & 45714.4 & 45857.7 & 46001.2 & 46144.8 & 46288.6 & 46432.5 \\
3.3 & 45000.0 & 45142.6 & 45285.3 & 45428.2 & 45571.2 & 45714.4 & 45857.7 & 46001.2 & 46144.8 & 46288.6 & 46432.5 \\
3.4 & 45000.0 & 45142.6 & 45285.3 & 45428.2 & 45571.2 & 45714.4 & 45857.7 & 46001.2 & 46144.8 & 46288.6 & 46432.5 \\
3.5 & 45000.0 & 45142.6 & 45285.3 & 45428.2 & 45571.2 & 45714.4 & 45857.7 & 46001.2 & 46144.8 & 46288.6 & 46432.5 \\
\hline$\widetilde{B C W S}(\alpha)$ & 268875.0 & 270375.8 & 271884.3 & 273400.5 & 274924.5 & 276456.3 & 277995.8 & 279543.0 & 281098.0 & 282660.8 & 284231.3 \\
\hline
\end{tabular}

Table 9 Results of fuzzy control

\begin{tabular}{|c|c|c|c|c|c|c|c|c|c|c|c|}
\hline \multirow{2}{*}{ Date $^{*}$} & \multirow{2}{*}{$\mathrm{ACWP}_{t}$} & \multicolumn{3}{|c|}{$\widehat{\operatorname{BCWP}}(0.7)_{t}$} & \multicolumn{4}{|c|}{$\widetilde{\mathrm{SV}}(0.7)_{t}$} & \multicolumn{3}{|c|}{$\widetilde{\mathrm{CV}}(0.7)_{t}$} \\
\hline & & $\operatorname{Si}(0.7)$ & $a_{2}$ & $\mathrm{Ss}(0.7)$ & $\operatorname{Si}(0.7)$ & $a_{2}$ & $a_{3}$ & $\mathrm{Ss}(0.7)$ & $\mathrm{Si}(0.7)$ & $a_{2}$ & $\mathrm{Ss}(0.7)$ \\
\hline $15 / 2 / 11$ & 9834.0 & 7053.7 & 7125.0 & 7302.4 & -2830.1 & -1405.0 & -25.0 & 331.2 & -2780.2 & -2709.0 & -2531.6 \\
\hline $28 / 2 / 11$ & 19316.0 & 15357.9 & 15513.0 & 15889.6 & -2268.1 & -352.0 & 6548.0 & 7886.8 & -3958.1 & -3803.0 & -3426.4 \\
\hline $15 / 3 / 11$ & 27986.0 & 27116.1 & 27390.0 & 28037.3 & -18387.1 & -13190.0 & -4285.0 & 102.3 & -869.9 & -596.0 & 51.3 \\
\hline $31 / 3 / 11$ & 67415.0 & 66115.5 & 66753.0 & 67908.4 & -17500.7 & -10607.0 & 3818.0 & 11923.5 & -1299.5 & -662.0 & 493.4 \\
\hline $15 / 4 / 11$ & 99490.0 & 97637.2 & 98553.0 & 99946.9 & -16731.9 & -9072.0 & 5978.0 & 14726.2 & -1852.8 & -937.0 & 456.9 \\
\hline $30 / 4 / 11$ & 132415.0 & 128742.6 & 129933.0 & 131562.2 & -5867.7 & 3308.0 & 16288.0 & 27988.8 & -3672.4 & -2482.0 & -852.8 \\
\hline $15 / 5 / 11$ & 152115.0 & 142421.9 & 143733.0 & 145465.7 & -16238.9 & -6642.0 & 2133.0 & 13635.8 & 706.9 & 2018.0 & 3750.7 \\
\hline $31 / 5 / 11$ & 170665.0 & 177690.6 & 179313.0 & 181312.6 & -9635.5 & 438.0 & 5763.0 & 20171.0 & 1575.6 & 3198.0 & 5197.6 \\
\hline $15 / 6 / 11$ & 199765.0 & 196127.8 & 197913.0 & 200052.1 & -17498.8 & -5712.0 & -4587.0 & 8495.2 & -3637.2 & -1852.0 & 287.1 \\
\hline $30 / 6 / 11$ & 231965.0 & 226162.7 & 228213.0 & 230579.3 & -13166.7 & -162.0 & -162.0 & 13389.5 & -5802.3 & -3752.0 & -1385.7 \\
\hline $15 / 7 / 11$ & 254215.0 & 251320.6 & 253593.0 & 256149.7 & -12994.2 & 468.0 & 468.0 & 14549.5 & -2894.4 & -622.0 & 1934.7 \\
\hline $31 / 7 / 11$ & 274815.0 & 270055.2 & 272493.0 & 275191.4 & -3345.3 & 3618.0 & 3.618 .0 & 11511.1 & -4759.8 & -2322.0 & 376.4 \\
\hline
\end{tabular}

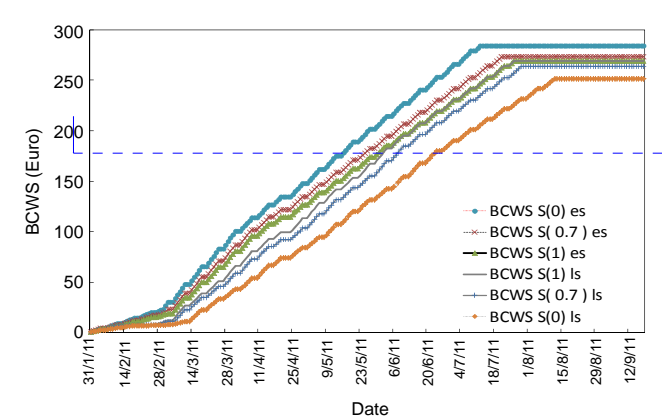

Fig. $12 S$-curves of the fuzzy BCWS traditional methodology) differ in magnitude and sign from the adjacent values $a_{3}$, enabling the project manager to report the real project performance in linguistic terms. Identical analysis can be performed on the resulting values for the cost variation $(\widetilde{\mathrm{CV}}(0.7))$, where column $a_{2}$ corresponds to the values given by the traditional methodology, and is adjacent to the range of permissible deviation values.

The ES, the progress-delays and the fuzzy linguistic interpretation for each of the scheduled controls are shown in Table 11. The column $a_{2}$

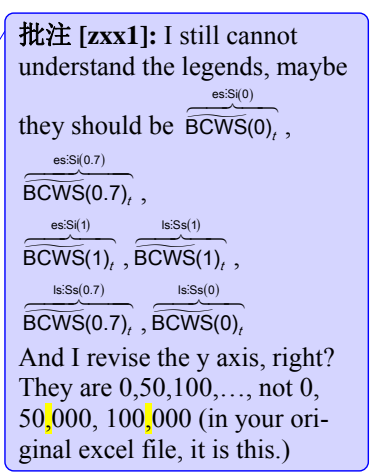


Table 10 Linguistic terms of fuzzy control for $\widetilde{\mathrm{SV}}(0.7)_{t}$ and $\widetilde{\mathrm{CV}}(0.7)_{t}$

\begin{tabular}{|c|c|c|c|c|}
\hline \multirow{2}{*}{ Date } & \multicolumn{2}{|r|}{$\widetilde{\mathrm{SV}}(0.7)_{t}$} & \multicolumn{2}{|c|}{$\widetilde{\mathrm{CV}}(0.7)_{t}$} \\
\hline & Scenario & Fuzzy analysis & Scenario & Fuzzy analysis \\
\hline $15 / 2 / 11$ & $\mathrm{D}$ & Slightly behind schedule & $\mathrm{E}$ & Over budget \\
\hline $28 / 2 / 11$ & $\mathrm{C}$ & On schedule & $\mathrm{E}$ & Over budget \\
\hline $15 / 3 / 11$ & $\mathrm{D}$ & Slightly behind schedule & $\mathrm{D}$ & Slightly over budget \\
\hline $31 / 3 / 11$ & $\mathrm{C}$ & On schedule & $\mathrm{D}$ & Slightly over budget \\
\hline $15 / 4 / 11$ & $\mathrm{C}$ & On schedule & $\mathrm{D}$ & Slightly over budget \\
\hline $30 / 4 / 11$ & $\mathrm{~B}$ & Slightly ahead of schedule & $\mathrm{E}$ & Over budget \\
\hline $15 / 5 / 11$ & $\mathrm{D}$ & Slightly behind schedule & A & Under budget \\
\hline $31 / 5 / 11$ & $\mathrm{~B}$ & Slightly ahead of schedule & A & Under budget \\
\hline $15 / 6 / 11$ & $\mathrm{D}$ & Slightly behind schedule & $\mathrm{D}$ & Slightly over budget \\
\hline $30 / 6 / 11$ & $\mathrm{D}$ & Slightly behind schedule & $\mathrm{E}$ & Over budget \\
\hline $15 / 7 / 11$ & B & Slightly ahead of schedule & $\mathrm{D}$ & Slightly over budget \\
\hline $31 / 7 / 11$ & $\mathrm{~B}$ & Slightly ahead of schedule & $\mathrm{D}$ & Slightly over budget \\
\hline
\end{tabular}

* Day/Month/Year

Table 11 Linguistic terms of $\widehat{\operatorname{ESch}}_{t}$ progress and delays

\begin{tabular}{|c|c|c|c|c|c|c|c|c|c|c|c|}
\hline \multirow{2}{*}{ Date $^{*}$} & \multirow{2}{*}{$p$} & \multicolumn{4}{|c|}{$\widetilde{\mathrm{ESch}}(0.7)_{p}$} & \multicolumn{4}{|c|}{$\widetilde{\mathrm{ESch}}(0.7)_{p}-p$} & \multirow{2}{*}{ Scenario } & \multirow{2}{*}{ Fuzzy analysis } \\
\hline & & $\operatorname{Si}(0.7)$ & $a_{2}$ & $a_{3}$ & $\mathrm{Ss}(0.7)$ & $\operatorname{Si}(0.7)$ & $a_{2}$ & $a_{3}$ & $\operatorname{Ss}(0.7)$ & & \\
\hline $15 / 2 / 11$ & 16 & 10.7 & 14.3 & 15.8 & 18.9 & -5.3 & -1.7 & -0.2 & 2.9 & $\mathrm{D}$ & Slightly behind time \\
\hline $28 / 2 / 11$ & 29 & 24.4 & 28.6 & 36.1 & 37.6 & -4.6 & -0.4 & 7.1 & 8.6 & $\mathrm{C}$ & On time \\
\hline $15 / 3 / 11$ & 44 & 36.2 & 37.7 & 42.2 & 44.0 & -7.8 & -6.3 & -1.8 & 0 & $\mathrm{D}$ & Slightly behind time \\
\hline $31 / 3 / 11$ & 60 & 52.4 & 56.5 & 63.3 & 66.2 & -7.6 & -3.5 & 3.3 & 6.2 & $\mathrm{C}$ & On time \\
\hline $15 / 4 / 11$ & 75 & 66.4 & 71.2 & 79.5 & 87.6 & -8.6 & -3.8 & 4.5 & 12.6 & $\mathrm{C}$ & On time \\
\hline $30 / 4 / 11$ & 90 & 86.7 & 92.4 & 98.5 & 102.9 & -3.3 & 2.4 & 8.5 & 12.9 & B & Slightly ahead of time \\
\hline $15 / 5 / 11$ & 105 & 94.2 & 100.2 & 105.9 & 112.9 & -10.8 & -4.8 & 0.9 & 7.9 & $\mathrm{C}$ & On time \\
\hline $31 / 5 / 11$ & 121 & 114.9 & 121.2 & 123.0 & 129.9 & -6.1 & 0.2 & 2.0 & 8.9 & B & Slightly ahead of time \\
\hline $15 / 6 / 11$ & 136 & 126.7 & 133.5 & 134.1 & 141.6 & -9.3 & -2.5 & -1.9 & 5.6 & $\mathrm{D}$ & Slightly behind time \\
\hline $30 / 6 / 11$ & 151 & 143.2 & 150.9 & 150.9 & 161.0 & -7.8 & -0.1 & -0.1 & 10.0 & $\mathrm{D}$ & Slightly behind time \\
\hline $15 / 7 / 11$ & 166 & 158.3 & 168.2 & 168.2 & 176.6 & -7.7 & 2.2 & 2.2 & 10.6 & B & Slightly ahead of time \\
\hline $31 / 7 / 11$ & 182 & 170.5 & 185.0 & 185.0 & 185.0 & -11.5 & 4.0 & 4.0 & 4.0 & B & Slightly ahead of time \\
\hline
\end{tabular}

corresponds to the 'membership equals one' of the fuzzy number (the same as offered by the traditional methodology); the column $a_{3}$ is produced as a result of the differences between the earliest and the latest times, and is adjacent to the lower $(\mathrm{Si}(0.7))$ and upper $(\mathrm{Ss}(0.7))$ limits of permissible deviation values in accordance with the established $\alpha$-cut.

\section{Conclusions}

The EVM is an integrated management control technique for assessing, measuring, understanding and quantifying project performance and progress in an objective manner, combining measurements of scope, production, and cost in an integrated system. In this paper, a comprehensive and robust approach for the fuzzy scheduling and earned value management of projects is presented. All the feasible schedules, between the earliest and the latest times, and the vagueness or imprecision in the estimated values, are taken into account.

The mathematical model incorporates modifications and improvements on previous contributions, and the treatment of the fuzzy EV management and the earned schedule is completely developed. Performance indexes have not been addressed, because they do not represent effectively the true development of the project. This leads to large deviations at the beginning of projects, and provides useful and rele- 
vant information only at the final stages of its implementation. The fuzzy EV proposed specifically includes: (1) Timing of the BCWS for each project period; (2) Consideration of every feasible project schedule between its earliest and latest times; (3) Formulation of SV, CV and especially ES; (4) Expression of results in linguistic and quantitative terms, adjusting the vagueness of the environment by changing the values established for the $\alpha$-cut; (5) Practical implementation of the mathematical model in a construction project with all the fuzzy values, demonstrating the feasibility of its use in complex projects.

The application of fuzzy logic to the scheduling and control of projects provides more realistic results that do not always coincide with those offered by the traditional approach. The findings of this study have a number of important implications for future practice, although further experimental research is necessary to establish the proper $\alpha$-cut value, as close as possible to reality, to improve the management of risks in construction projects.

This contribution is not a tool to be applied in a direct and simple way by practitioners, but aims to serve as a starting point for specialists in order to develop user-friendly and practical computer applications that provide solutions for the problems of uncertainty in project management. This proposed approach may serve to develop software that could be put on the market and used by practitioners in the subject and project managers. This software can be implemented in commercial computer applications as well known and easy to use as Microsoft $^{\mathcal{O}}$ Excel $^{\mathcal{O}}$; this is one of the lines of research that the authors are currently pursuing.

\section{Acknowledgements}

The authors want to thank Ms. Doria GIL-SENABRE, Universitat Politècnica de València, Spain, for the support provided.

\section{References}

Anbari, F.T., 2003. Earned value project management: Methods and extensions. Project Management Journal, 34(4):12-23.

Bonnai, P., Gourc, D., Lacoste, G., 2004. Where do we stand with fuzzy project scheduling? Journal of Construction
Engineering and Management, 130(1):114-123.

Buckley, J.J., Eslami, E., Feuring, T., 2002. Fuzzy mathematics in Economics and Engineering: Studies in Fuzziness and Soft Computing. Physica-Verlag, Heidelberg (Germany).

Chanas, S., Kambourowski, J., 1981. The use of fuzzy variables in PERT. Fuzzy Sets and Systems, 5(1):11-19. [doi:10.1016/0165-0114(81)90030-0]

Chen, C.T., Huang, S.F., 2007. Applying fuzzy method for measuring criticality in project network. Information Sciences, 177:2448-2458. [doi:10.1016/j.ins.2007.01.035]

Fleming, Q.W., Koppelman, J.M., 1998. Earned value project management. The Journal of Defense Software Engineering, 16(1):19-23.

Kuchta, D., 2005. Fuzzyfication of the earned value method. WSEAS Transactions on Systems, 4(12):2222-2237.

Laviolette, M., Seaman, J.W., Barrett, J.D., Woodall, W.H., 1995. A probabilistic and statistical view of fuzzy methods. Technometrics, 37(3):249-261.

Lawry, J., 2001. An alternative approach to computing with words. International Journal of Uncertainty, Fuzziness and Knowledge-Based Systems, 9:3-16. [doi:10.1142] S0218488501000958]

Lipke, W., 2003. Schedule is Different. The Measurable News, March \& Summer, p.31-34. Available from http://www.earnedschedule.com/Docs/Schedule $\% 20$ is $\%$ 20Different.pdf [Accessed on May 20, 2011].

Lipke, W., Zwikael, O., Henderson, K., Anbari, F.T., 2009. Prediction of project outcome: The application of statistical methods to earned value management and earned schedule performance indexes. International Journal of Project Management, 27(4):400-407. [doi:10.1016/ j.ijproman.2008.02.009]

Liu, Y.C., Yang, S.M., Lin, Y.T., 2010. Fuzzy finish time modeling for project scheduling. Journal of Zhejiang University-SCIENCE A (Applied Physics \& Engineering), 12(11):946-952. [doi:10.1631/jzus.A10001115]

Moslemi, N.L., Salehipour, A., 2011. Evaluating fuzzy earned value indices and estimates by applying alpha cuts. Expert Systems with Applications, 38(7):8193-8198. [doi:10.1016/j.eswa.2010.12.165]

Moslemi, N.L., Shadrokh, S., Salehipour, A., 2011. A fuzzy approach for the earned value management. International Journal of Project Management, 29(6):764-772. [doi:10.1016/j.ijproman.2010.07.012]

Noori, S., Bagherpour, M., Zareei, A., 2008. Applying fuzzy control charts in earned value analysis: A new application. World Applied Sciences Journal, 3(4):684-690.

Pellicer, E., Pellicer, T.M., Catalá, J., 2009. An integrated control system for SMEs in the construction industry. Revista de la Construcción, 8(2):4-17.

Ponz-Tienda, J.L., 2010. Robust GRCPSP based on Production Processes for Construction Projects. PhD Thesis, Universitat Politècnica de València, Valencia (in Spanish).

Prade, H., 1979. Using fuzzy set theory in a scheduling problem: A case study. Fuzzy Sets and Systems, 2(2):153-165. [doi:10.1016/0165-0114(79)90022-8]

Shipley, M., Korvin, A., Omer, K., 1996. A fuzzy logic ap- 
proach for determining expected values: A project management application. The Journal of the Operational Research Society, 47(4):562-569.

Wang, J., Hao, J., 2007. Fuzzy linguistic PERT. IEEE Transactions on Fuzzy Systems, 15(2):133-144. [doi:10.1109/ TFUZZ.2006.879975]

Yao, J.S., Wu, K.M., 2000. Ranking fuzzy numbers based on decomposition principle and signed distance. Fuzzy Sets and Systems, 116(2):275-288. [doi:10.1016/S0165-0114 (98)00122-5]
Zadeh, L.A., 1965. Fuzzy sets. Information and Control, 8(3):338-353. [doi:10.1016/S0019-9958(65)90241-X]

Zadeh, L.A., 1975. The concept of a linguistic variable and its application to approximate reasoning. Part I. Information Sciences, 8(3):199-249. [doi:10.1016/0020-0255(75) 90036-5]

Zadeh, L.A., 1995. Discussion: Probability theory and fuzzy logic are complementary rather than competitive. Technometrics, 37(3):271-276. 